\title{
Philosophiques
}

\section{Les cours de Marbourg et la phénoménologie}

\section{Denis Fisette}

Volume 20, numéro 2, automne 1993

Perspectives sur la phénoménologie et l'intentionnalité

URI : https://id.erudit.org/iderudit/027228ar

DOI : https://doi.org/10.7202/027228ar

Aller au sommaire du numéro

Éditeur(s)

Société de philosophie du Québec

ISSN

0316-2923 (imprimé)

1492-1391 (numérique)

Découvrir la revue

Citer cet article

Fisette, D. (1993). Les cours de Marbourg et la phénoménologie. Philosophiques, 20(2), 303-321. https://doi.org/10.7202/027228ar d'utilisation que vous pouvez consulter en ligne.

https://apropos.erudit.org/fr/usagers/politique-dutilisation/ 


\title{
IES COURS DE MARBOURG ET LA PHÉNOMÉNOLOGIF*
}

\author{
par \\ Denis Fisette
}

La phénoménologie husserlienne, faut-il le rappeler, a marqué d'une manière significative la réflexion de Heidegger en philosophie. Les textes sont d'ailleurs nombreux où il reconnaît sa dette ${ }^{\mathrm{I}}$. Sein und Zeit, ouvrage dédié à Husserl, est le fruit d'une longue collaboration avec Husserl, collaboration qui s'est poursuivie avec la rédaction commune du fameux article destiné à l'Encyclopæedia Britannica et avec l'édition par Heidegger des Leçons pour une phénoménologie de la conscience intime du temps ${ }^{2}$ de Husserl.

Cependant, les remarques trop souvent cursives de ces textes publiés du vivant de Heidegger sont significatives mais tout à fait insuffisantes lorsqu'il s'agit d'établir avec quelque précision ce qui, de la phénoménologie husserlienne, a pu contribuer à l'élaboration de Sein und Zeit ${ }^{3}$. Les cours de Marbourg $\left(1925^{-29}\right)^{4}$, contemporains de Sein und Zeit, contiennent un précieux complément d'information sur la conception de la phénoménologie que

I. Il s'agit principalement des textes regroupés dans la troisième partie de Questions IV, Paris, Gallimard, 1976.

2. M. Heidegger, « Seconde version de l'article "Phenomenology", 1927 », dans Heidegger, Paris, l'Herne, 1983 ; E. Husserl, Leçons pour une phénoménologie de la conscience intime du temps, Paris, P.U.F., 1964.

3. M. Heidegger, Sein und Zeit, Tübingen, M. Niemeyer, 1979; tr. fr. par E. Martineau, Être et Temps, Paris, Authentica, 1985.

4. M. Heidegger, Prolegomena zur Geschichte des Zeitbegriffs, Gesamtausgabe Bd. zo, Frankfurt, Klostermann, 1979; Grundprobleme der Phänomenologie, Gesamtausgabe Bd. 24, Frankfurt, Klostermann, 1975. tr. fr. Les problèmes fondamentaux de la phénoménologie, Paris, Gallimard, 1985 ; Metaphysische Anfangsgründe der Logik im Ausgang von Leibniz, Gesamtausgabe Bd. 26, Frankfurt, Klostermann, I978.

- Une partie de ce texte a fait l'objet d'une conférence dans le cadre du congrès de l'Association Canadienne de Philosophie à l'université de Carleton au mois de mai dernier. Je remercie P. Buckley qui l'a commenté et le CRSH pour son soutien financier. 
Heidegger attribue à Husserl. Nous pensons en particulier à Prolegomena zur Geschichte des Zeitbegriffs dans lequel Heidegger introduit la doctrine de Sein und Zeit en partant d'une évaluation des apports de la phénoménologie husserlienne. Heidegger ${ }^{5}$ y attribue trois découvertes décisives à la phénoménologie de Husserl : l'intentionnalité, l'intuition catégoriale et le sens originaire de l'apriori. La première découverte précise le genre de choses dont doit s'occuper la philosophie, son champ thématique, à savoir la conscience intentionnelle et, comme nous le préciserons tantôt, les corrélations noético-noématiques. La deuxième découverte, dont Heidegger dira plus tard dans « Mon chemin de pensée et la phénoménologie ${ }^{6}$ qu'elle fut déterminante pour la question du sens de l'être, concerne la distinction dégagée dans la sixième des Recherches logiques ${ }^{7}$ entre « intuition catégoriale » et « intuition sensible». Elle lui aurait permis, en outre, de surmonter les difficultés liées à la conception traditionnelle des catégories et d'ouvrir la possibilité de constituer une « ontologie scientifique ». Finalement, le sens originaire de l'apriori, ce qu'il appelle également le « lieu du transcendantal », est précisément la dimension et le niveau de ce dont il est question en phénoménologie. Encore ici, Heidegger attribue à Husserl le mérite d'avoir montré, contre Kant, que l'apriori n'est ni de l'ordre du subjectif, ni de l'étant, mais qu'il appartient à la dimension de « l'être de l'étant $»^{8}$. Avec le champ thématique, la perspective et la méthode, sont mis en place les éléments essentiels que Heidegger fait intervenir dans șa définition de la phénoménologie:

Phänomenologie ist analytische Deskription der Intentionalität in ihrem Aprioris?

Avec cette définition de la phénoménologie husserlienne, nous disposons de tous les éléments nécessaires pour poser la

5. M. Heidegger, Prolegomena zur Geschichte der Zeitbegriffs, op. cit, p. 34.

6. M. Heidegger, « Mon chemin de pensée et la phénoménologie » dans Questions IV, Paris, p. 168. Sur cette question, on pourra consulter l'article de J. Taminiaux, « Le regard et l'excédent : remarques sur Heidegger et les Recherches logiques », Revue philosophique de Louvain, 75, 1977, p. 74-100.

7. E. Husserl, Recherches Logiques, volume III, Paris, P.U.F., 1969.

8. M. Heidegger, Prolegomena zur Geschichte der Zeitbegriffs, op. cit., p. IоI.

9. Ibid. p. 108, 129 . 
question qui fera l'objet de mon investigation : dans quelle mesure ces trois découvertes fondamentales que Heidegger attribue à la phénoménologie husserlienne ont-elles contribué à l'élaboration de la question du sens de l'être dans Sein und Zeit?

Cette question, comme on le sait, est à maints égards périlleuse. Elle indignera certes ceux qui, fidèles à Heidegger, ne fréquentent la phénoménologie husserlienne, et n'y trouvent d'intérêt, que dans l'horizon d'une philosophie en rupture avec la tradition. Mais elle irritera à coup sûr ceux qui, inquiets du destin de la phénoménologie après Husserl, et ardents défenseurs de l'idéal d'une philosophie comme «science rigoureuse », se méfient des effets trop souvent rhétoriques d'une philosophie des profondeurs. C'est le cas de Husserl qui, dans les notes en marge de son exemplaire de Sein und Zeit, déplorant le « discours éthico-théologique », n'a vu dans cette entreprise que la «traduction » de sa propre phénoménologie «dans l'ordre de l'anthropologie ». À l'extrême opposé, nous trouvons J. Beaufret ${ }^{\mathrm{TO}}$ qui parle d'un « éclectisme » de la relation Husserl et Heidegger voulant par là accentuer la rupture quant à la nature et au style de questionnement. Autrement, il n'a sans doute pas tort de parler d'éclectisme. Le commentaire récent de la première partie de Sein und Zeit par H. Dreyfus ${ }^{\text {II }}$ va essentiellement dans le même sens qui soutient que cet ouvrage représente en fait une critique dévastatrice de la doctrine husserlienne de l'intentionnalité même si Heidegger fait rarement allusion à cette dernière dans Être et Temps. Cette thèse avait déjà été proposée comme hypothèse de travail aux rencontres de l'Institut d'été sur « Les perspectives analytiques et continentales sur l'intentionnalité $»^{12}$ qui se sont tenues entre 1980 et 1983 à Berkeley et auxquelles ont pris part des figures aussi importantes que J. Haugeland, J. Searle, D. Føllesdal, R. van Gulick, D.W. Smith, R. McIntyre, etc ${ }^{\text {I3 }}$. Ces

Io. J. Beaufret, « Préface » à M. Heidegger, Le Principe de raison, Paris, Gallimard, ig6a. p. 25.

II. H. Dreyfus, Being-in-the-World, Cambridge, M.I.T. Press, 1991.

12. Le programme de ces rencontres a été publié sous le titre Phenomenology and Existentialism, The Council for Philosophical Studies, San Franscisco State University, ig81.

13. Ces rencontres ont donné lieu à d'importantes publications, notamment 
rencontres sont également à l'origine de plusieurs débats qui nourissent ce qu'on appelle aux USA la « west coast phenomenology » et qui portent notamment sur l'opposition apparente de Husserl et Heidegger sur la phénoménologie. Un passage d'un article récent ${ }^{\mathrm{I4}}$ de Dreyfus en résume brillamment les enjeux :

Ce qui ne va pas avec toute la doctrine du noème dans les Idées directrices I...I, doctrine suivant laquelle la pensée est dissociable du monde et dirigée vers ce dernier uniquement au moyen de son contenu intentionnel, c'est qu'elle ne rend pas justice à l'habileté de l'agir quotidien. Telle fut la raison pour laquelle Heidegger remplaça la phénoménologie transcendantale par la phénoménologie existentiale. Heidegger reconnaît que la directionalité est essentielle à l'activité humaine mais il conteste que toute directionalité soit psychique. Il cherche a montrer que le commerce de l'agir quotidien est le mode premier de l'activité humaine et que les états mentaux ayant un contenu intentionnel ne sont pas nécessaires pour qu'un tel commerce ait lieu et parce que tout agir dirigé vers quelque chose présuppose un arrière-plan de familiaritè quotidienne dont on ne peut rendre compte en termes intentionnels. C'est ainsi que Heidegger rejette la possibilité du solipsisme méthodologique et de la réduction transcendantale. Il argue que même lorsque nous agissons d'une manière délibérée [...], notre pensée ne peut être dirigée vers quelque chose sinon à partir d'un arière-plan de pratiques sociales partagées.

Ce passage, comme plusieurs autres qui abondent essentiellement dans le même sens, montrent que l'enjeu principal de cette critique repose sur la conception de l'intentionnalité qu'il attribue à Husserl ${ }^{15}$. La stratégie de Dreyfus consiste, d'une part, à rapprocher la conception husserlienne de l'intentionnalité de la théorie représentationnelle de l'esprit, théorie qu'il fait remonter à Descartes et dont la relation sujet / objet serait caractéristique; et ce, d'autre part, afin de faire valoir la critique heideggerienne de ce néo-cartésianisme. Dans ce qui suit, j' examinerai les enjeux sousjacents à l'usage qu'il fait de la philosophie heideggerienne de Sein

l'anthologie de H. Dreyfus (dir.), Husserl, Intentionality, and Cognitive Science, Cambridge, M.I.T. Press, I982 ; D. W. Smith et R. McIntyre, Husserl and Intentionality, Dordrecht, Reidel, 1982 ; J. Searle, Intentionality, Cambridge, Cambridge U.P., I983; J. Haugeland (dir.), Mind Design, Cambridge, M.I.T. Press, 1981.

14. H. Dreyfus « The Mind dans Husserl » TL.S., July 12, 1991, p. 25. Dreyfus s'en prend ici au livre de D. Bell, Husserl, London, Routhledge, 1990.

15. Cest également l'objet du débat qui l'oppose à R. McIntyre dans une sērie d'articles parus en français dans un numéro spécial de la revue Etudes Philosophiques. I, I99I. 
und Zeit dans sa critique Husserl. Je m'en tiendrai aux thèmes suivants : a) La « critique » par Heidegger de la phénoménologie husserlienne ; b) Le noème husserlien et les deux dogmes de la théorie traditionnelle de l'intentionnalité ; c) L'aptitude de la théorie husserlienne de l'intentionnalité à rendre compte de la dimension, de l'agir sous-jacente au Dasein ; d) finalement, nous examinerons les relations complexes qu'entretiennent les concepts d'intentionnalité et de transcendance.

I

Rappelons tout d'abord que par «Kritik», Heidegger vise moins à jeter un discrédit sur une doctrine que la « libération de la chose et de la compréhension ${ }^{16}$. Libérer la chose et sa compréhension revient à faire de la maxime de la phénoménologie, « zu den Sachen selbst », un principe - principe que Husserl a lancé contre toute entreprise visant à ériger les sciences positives et régionales au rang de philosophie première. Or la « critique » principale qui est adressée à la phénoménologie husserlienne a trait à la radicalité de ce principe et elle porte sur l'idée traditionnelle de philosophie dont Husserl aurait hérité du cartésianisme. Ainsi, même lorsqu'il s'agit de la détermination de son thème le plus propre, l'intentionnalité, la phénoménologie reste marquée par la philosophie de la conscience. C'est qu'en définissant la chose même comme conscience intentionnelle, Husserl aurait négligé la question de l'être de l'intentionnel et, plus généralement, la question du sens de être qui, selon Heidegger, constitue le champ primordial de la recherche phénoménologique. C'est pourquoi Heidegger peut écrire :

Phänomenologie ist daher in der Grundaufgabe der Bestimmung ihres eigensten Feldes unphänomenologisch ! - das heißt vermeintlich phänomenologisch ${ }^{17}$ !

I6. M. Heidegger Prolegomena, $\S$ II, p. I40 et sq. Comparer avec ce que Heidegger appelle dans Kant et le probleme de la métaphysique, Paris. Gallimard, 1953, «Wiederholung » (répétition) : « Nous entendons par répétition d'un probleme fondamental la mise au jour des possibilités quil recèle. La mise au jour de celles-ci a pour effet de transformer le problème considéré et, par là même, de lui conserver son contenu authentique. » P. $26 \mathrm{I}$.

17. M. Heidegger, Prolegomena zur Geschichte der Zeitbegriffs, op.cit., p. 35. 
La phénoménologie husserlienne, même lorsqu'il s'agit de son thème le plus propre, l'intentionnalité, n'est en fait que prétendument phénoménologique puisqu'elle reste marquée par l'héritage cartésien de ce qu'on pourrait appeler une « ontologie de la conscience $\gg$. Une phénoménologie conséquente avec la radicalité de son principe devra naturellement ouvrir sur une ontologie du « Dasein ». Mais la structure intentionnelle en tant que telle ne semble pas être mise en cause dans ce projet de radicalisation puisque, comme le soutient Heidegger dans son séminaire de Zähringen ${ }^{18}$, « penser l'intentionnalité à fond, c'est la situer dans l'ekstatique du Dasein ». Or la dimension ekstatique et existentiale que Heidegger prétend ouvrir avec son concept de Dasein n'est pas sans rapport avec l'intentionnalité :

L'intentionnalité appartient à l'existence du Dasein. Pour le Dasein, en même temps que son existence, se trouvent toujours déjà dévoilés, d'une certaine manière, un étant et une connexion avec de l'étant, sans que celui-ci soit à proprement parler objectivé. Exister signifie donc aussi être auprès de l'étant en se comportant par rapport à lui ${ }^{19}$.

Comment Heidegger a-t-il pu parler d'une « découverte » de l'intentionnalité par Husserl puisque, comme on le sait, c'est Brentano qui l'avait introduite dans sa Psychologie d'un point de vue empirique ? De la réponse à cette question dépend la possibilité de dissocier l'intentionnalité de la conscience et de la relation sujetobjet à laquelle elle avait èté assujettie dans la tradition cartésienne. Mon hypothèse de travail est que Heidegger a clairement démarqué dans ses cours de Marbourg ce qu'il qualifie de « conception traditionnelle » de l'intentionnalité qu'il attribue par ailleurs à Brentano, de la doctrine husserlienne. Si on admet cela, et en supposant que les critiques adressées par Heidegger à la phénoménologie husserlienne visent moins sa doctrine de l'intentionnalité que l'interprétation idéaliste qu'il lui a donnée dans le premier livre des Idées directrices ${ }^{20}$, il serait alors illégitime de

I8. M. Heidegger, art. cit., P 472.

19. M. Heidegger, Les Problemes fondamentaux de la phénoménologie, op. cit., p. 195.

20. E. Husserl, Idées directrices pour une phénoménologie et une philosophie phénoménologique pures, tr. fr. par P. Ricoeur, Paris, Gallimard, I950. 
détourner vers la doctrine husserlienne les reproches et contresens qui visent en fait la conception traditionnelle de l'intentionnalité.

Partons une fois de plus du fameux passage de la Psychologie d'un point de vue empirique dans lequel Brentano introduit ce qui allait devenir la définition standard de l'intentionnalité au vingtième siècle :

Ce qui caractérise tout phénomène psychique, c'est ce que les Scolastiques du moyen âge ont appelé l'inexistence intentionnelle (ou encore mentale) et ce que nous pourrions appeler nous-mêmes [...] rapport à un contenu, direction vers un objet (sans qu'il faille entendre par là une réalitél ou objectivité immanente ${ }^{2 I}$.

Comme le remarque Heidegger ${ }^{22}$, le concept d'intentionnalité est introduit en réponse à la question de savoir ce qui distingue la nature des phénomènes psychiques (audition par exemple) de celle des phénomènes physiques (un son). Par la fonction qui lui est assignée, ce concept réintroduit une dichotomie entre ce qui est de l'ordre du physique, de la res extensa ou de l'objet, et ce qui est propre à la nature des phénomènes psychiques, à savoir le caractère spécifique de la res cogitans ou du sujet.Ces derniers se caractérisent par l'idée de directionalité [sich richten aufl, l'idée que toute représentation est représentation de quelque chose, est dirigée vers quelque chose. Maintenant, cette conception de l'intentionnalité comme directionalité est doublée chez le premier Brentano de sa doctrine de «l'inexistence intentionnelle », doctrine suivant laquelle tout acte « contient quelque chose en soi à titre d'objet », quelque chose qui inexiste intentionnellement dans l'acte. De cela découle sa définition des phénomènes psychiques comme étant ceux «qui contiennent intentionnellement un objet [Gegenstand] en eux $^{23}$. »

Mais que signifie ici « objet intentionnel »? Comme le remarquait déjà Heidegger dans ses cours de Marbourg ${ }^{24}$, la première théorie de Brentano n'a jamais réussi à préciser d'une

\footnotetext{
21. F. Brentano, La psychologie d'un point de vue empirique, tr. fr. par M. de Gandillac, Paris, Aubier, 1944, p. 102.

22. M. Heidegger, Prolegomena, p. 26.

23. F. Brentano, op cit., p. 102.

24. M. Heidegger, Prolegomena, p. 6I-62.
} 
manière convaincante si ce quelque chose auquel tout acte intentionnel se rapporte, est l'étant lui-même ou simplement la manière dont il est conçu. Et comme le reconnaîtra plus tard Brentano lui-même, c'est qu'il ne disposait pas alors de la distinction entre l'intentio (noèse) et l'intentum (noème) que l'un de ses étudiants les plus perspicaces introduira plus tard. Nous verrons que Husserl lui reproche également d'identifier l'intentionnalité au psychique.

Maintenant, dans les Problèmes fondamentaux de la phénoméno$\operatorname{logie} e^{25}$, Heidegger cherche à montrer que la théorie de Brentano n'est en fait qu'un cas particulier de ce qu'il denonce sous le titre de « relation sujet / objet». C'est que, comme nous l'avons noté, il aurait conçu l'intentionnalité « comme relation subsistante entre deux étants subsistants - un sujet psychique et un objet physique ${ }^{26}$. Ce qui caractérise cette conception de l'intentionnalité, c'est que toute relation ainsi comprise dépend de l'existence des termes de la relation. Il en découle deux « dogmes » que Heidegger associe avec la conception traditionnelle de l'intentionnalité.

I) A tout processus psychique correspond un objet réel ;

2) « La sortie hors de lui du psychique vers le physique ${ }^{27}$. »

Subordonner la relation intentionnelle à la relation sujet / objet, c'est commettre deux erreurs que Heidegger ${ }^{28}$ dénonce sous le titre « d'objectivation » et de « subjectivisation ». L'erreur d'objectivation est clairement attribuée à Brentano et elle consiste à faire dépendre l'intentionnalité d'un acte, ce qui est perçu dans un acte de perception par exemple, de l'existence de l'objet (perçu). Contre ce dogme, Heidegger fait valoir à plusieurs endroits que cette conception de l'intentionnalité ne peut rendre compte des cas où, victime d'une hallucination, aucun objet réel ne correspond à ma perception actuelle.

25. M. Heidegger, op.cit, p. 84.

26. Ibid.

27. Comparer avec les 2 présupposés de la conception traditionnelle de l'intentionnalité subordonnée à la relation sujet / objet dans Metaphysische Anfangsgründe der Logik im Ausgang von Leibniz : « Mit jedem Subjekt ist ein Objekt vorhanden (I) und mit jedem Objekt ein Subjekt (2) », p. I6I.

28. M. Heidegger, Les problèmes fondamentaux de la phénoménologie, p. 90. 
La relation intentionnelle ne résulte pas d'abord de l'être effectivement présent-subsistant des objets, mais elle est impliquée dans le percevoir lui-même, qu'il soit ou non entaché d'illusion. Il faut que le percevoir soit perception-de quelque chose laliquid], afin que je puisse me faire illusion sur quelque chose ${ }^{29}$.

L'argument de Heidegger m'apparaît tout à fait fondé contre ce type de conception représentationnelle et solipsiste qui ne peut minimalement rendre compte de la différence entre la perception d'un objet réel et une simple représentation ou image.

A l'erreur d'objectivation correspond le contre-sens subjectiviste lié au second dogme et à la conception du psychique comme une «boîte fermée », une monade sans fenêtre, dans laquelle le sujet n'aurait jamais affaire qu'à ses propres représentations. Mais comment alors le sujet pourrait-il sortir de sa sphère d'appartenance et entrer en relation avec le monde des objets? Il faut préciser que ce qui est dénoncé ici par le terme «subjectivisation » ce n'est pas que les actes, ou vécus intentionnels, « appartiennent à la sphère du sujet », mais bien plutôt que « ce sur quoi se dirigent ces vécus immanents soit lui aussi subjectif ${ }^{30}$. C'est ce que confirme Heidegger dans le passage suivant : « Le percevoir se dirige d'abord seulement sur le subjectif, c'est-à-dire sur les données sensorielles ${ }^{31}$. »

Encore ici, l'agent n'aurait jamais accès aux choses extérieures que par le biais d'une représentation ou image qui serait donnée immédiatement à la conscience. L'argument contre ce dogme est à nouveau la possibilité de l'erreur :

l...l le sens de l'illusion tient ici précisément à ce que, prenant l'arbre pour un homme, j'appréhende ce que je perçois et crois percevoir comme un étant subsistant. A travers cette illusion perceptive, c'est l'homme lui-même qui m'est donné et non point une représentation de l'homme ${ }^{32}$.

L'erreur qui est imputée ici à la doctrine du premier Brentano consiste en ceci que si dans la perception nous visons une représentation plutôt que la chose même, alors il devient impossible

\footnotetext{
29. Ibid., p. 85 .

30. Voir, par exemple, Les Problemes fondamentaux de la phénoménologie, op.cit., p. 87.

31. Ibid., p. 96-7.

32. Ibid., p. 88.
} 
de faire la différence entre la chose transcendante et une simple image.

La question est maintenant de savoir si la théorie husserlienne de l'intentionnalité est vulnérable à la critique de ce que Heidegger appelle la conception traditionnelle de l'intentionnalité. C'est ce que suggère entre autres Dreyfus. Pourtant, si on se fie aux cours de Marbourg, Heidegger attribue précisément à Husserl le mérite d'avoir su aller contre les deux dogmes de la conception traditionnelle de l'intentionnalité ${ }^{33}$. Et s'il a pu, après Brentano, parler d'une « découverte fondamentale » de l'intentionnalité par Husserl, c'est précisément parce que ce dernier a réussi à dissocier son concept de « relation intentionnelle » de la relation sujet / objet à laquelle Brentano l'avait assujettie. ${ }^{34}$ C'est ce que confirme aussi cette remarque de Heidegger dans les Prolégomènes ${ }^{35}$, où il fait valoir que l'argument principal de Husserl contre le «mentalisme » de Brentano repose sur la distinction entre 《 acte » ou « vécu psychique » et « contenu idéal », entre intentio et intentum pour employer la terminologie de Heidegger, ou, pour le dire dans les termes des Idées directrices, entre noesis et noema. Or, explique Heidegger ${ }^{36}$, Brentano ne disposait que de l'intentio ou de la noèse et c'est pourquoi il n'a pas réussi à surmonter les difficultés que nous venons d'évoquer. Heidegger suggère donc que c'est par l'introduction du concept de noème que Husserl vient à bout de cette forme de cartésianisme. L'intentionnalité n'est plus à concevoir comme une relation quelconque entre un sujet clos sur lui-même et un objet transcendant, mais bien comme «structure », et une structure qui informe l'ensemble de ce que nous étudierons maintenant sous le titre " comportements du Dasein ». Ce qui la caractérise, ce sont les corrélations noético-noématiques ou ce que Heidegger appelle la co-appartenance entre l'intentio et l'intentum. Et c'est précisément cette idée

33. M. Heidegger, Prolegomena zur Geschichte des Zeitbegriffs, op. cit., p. $4 \mathrm{I}$ et Metaphysische Anfangsgründe des Logik im Ausgang von Leibniz, p. 160 et sq.

34. Cest ce qu' on pourrait montrer en énumérant quelques traits caractéristiques que Heidegger attribue au concept relationnel d'intentionnalité. Je me contente pour le moment de renvoyer aux Prolegomena, p. 51 et sq.

35. Op.cit., p. 160 .

36. Ibid., p. $6 \mathrm{r}$. 
de corrélation qui fait de l'intentionnalité, aux yeux du philosophe de Marbourg, la découverte fondamentale de la phénoménologie husserlienne ${ }^{37}$.

\section{III}

Rappelons tout d'abord cette remarque tout à fait significative de Heidegger dans son cours de igzg où l'analyse du concept de « relation intentionnelle » constitue une des tâches préparatoires de Sein und Zeit ${ }^{38}$. Cependant, comme nous l'avons déjà indiqué, il faut également tenir compte du déplacement de la structure intentionnelle dans l'ekstatique du Dasein qui s'opère à l'époque de Sein und Zeit, déplacement qui exige un enrichissement et un élargissement de l'intentionnalité. Ainsi, à la corrélation intentio-intentum, il faut compter sur l'auto-compréhension - la relation à soi - qui est constitutive d'une relation intentionnelle ${ }^{39}$, et sur « la compréhension du mode d'être de ce qui est visé dans l'intentum $\gg{ }^{40}$. Ces deux nouvelles propriétés que Heidegger attribue à une relation intentionnel vont de pair avec la revalorisation de l'action non délibérée et quotidienne du Dasein et avec l'élargissement du champ de l'intentionnalité à l'ensemble des comportements IVerhaltungenl « quotidiens et pré-philosophiques » du Dasein. Le mot «Verhaltung » qui revient si souvent dans les cours de Marbourg et que l'on pourrait traduire par « agir »si l'on entend par là «comportement intentionnel ${ }^{4 \mathrm{I}}$, désigne alors

37. Dans son article « Transcendance et intentionnalité : Heidegger et Husserl sur les prolégomènes d'une ontologie phénoménologique », R. Bernet écrit en marge des Prolegomena zur Geschichte des Zeitbegriffs : « L'énigme de la relation intentionnelle n'est pas sa transitivité mais plutôt ce que Husserl appelle "la corrélation" entre intentio et intentum que Heidegger à son tour désigne comme leur "co-appartenance" (Mitzugehörigkeit, p. 63). Heidegger se fait ici le défenseur de ce que, plus tard, on a appelé une "phénoménologie noéma. tique", c'est-à-dire une tentative de s'approcher de la mystérieuse co-appartenance intentionnelle à partir d'un interrogatoire serré de lintentum (p. 48,60). Heidegger argue du caractère naturel d'une telle démarche qui se laisse porter par le mouvement de la relation intentionnelle vers l'intentum (J La description phénoménologique delintentum (p. 48-54) offreloccasion d'un premier questionnement sur la signification ontologique de l'intentionnalité, » P. 197.

38. M. Heidegger, Metaphysische Anfangsgründe der Logik im Suagang von Leibniz, op. cit., p. I64. Il s'agit des $\S \S 122^{-13}$ de Sein und Zeit.

39. M. Heidegger, Les problèmes fondamentaux de la phénoménologie, op. cit., p. 196.

40. Ibid., p. 97.

4I. Dans un cours de 1930 intitulé Les concepts fondamentaux de la métaphysique, Paris. 
la dimension de la praxis ou de l'agir reléguée au second plan par la métaphysique classique. C'est pourquoi il dénonce l'orientation unilatéralement épistémique de la phénoménologie de Ideen I qui semble accorder un privilège démesuré aux actes objectivants. Pour lui, l'attitude « contemplative » qui est à l'œuvre dans la phénoménologie de la perception n'est en fait qu'un mode dérivé de comportement.

Revenons maintenant à la lecture de Dreyfus qui voit dans ce « déplacement » l'indice d'une critique radicale de l'intentionnalité chez Husserl. A cet égard, il formule deux thèses qu'il attribue à Heidegger :

Heidegger will seek to show that $(\mathrm{I})$ intentionality without self-referential mental content is characteristic of the unimpeded mode of Dasein's everyday activity, whereas mental-state intentionality is a derivative mode, and (2) both these modes of directedness (ontic transcendence) presuppose being-in-theworld, a more originary transcendence ${ }^{42}$.

Ces deux thèses se transforment rapidement en objections à l'endroit de Husserl :

Les deux objections se rapportent à une description du comportement usuel cherchant à montrer que, contrairement à ce que nous disent la psychologie ordinaire et la tradition philosophique, on ne peut pas comprendre l'action en termes d'une sphère immanente au sujet et contenant des représentations qui se référeraient avec ou sans succès à un objet transcendant. C'est cette théorie de l'action qui permet à Husserl et à Fodor de supposer qu'ils peuvent mettre l'existence entre parenthèses et de décrire une sphère autosuffisante du contenu intentionnel. [... $]^{43}$.

On le voit, en interprétant le noème sur le modèle de la théorie représentationnelle de l'esprit, Dreyfus conçoit la réduction transcendantale comme une forme de « solipsisme méthodologique » et il n'a pas de mal à montrer que, dans cette

Gallimard, 1992, Heidegger accorde beaucoup d'importance, au concept de Verhalten qu'il distingue de celui de Benehmen, lesquels désignent deux modes d'être différents : « Ia façon dont est lhomme, nous l'appelons Verhalten ; la façon dont est l'animal, nous lappelons Benehmen [J. Le comportement animal n'est pas une façon de faire et d'agir, comme l'est le Verhalten humain, mais c'est un mouvement pulsionnel [Treibenl, », P. 347. Le Verhalten ne peut être dit intentionnel que parce qu'il a la structure du « en tant que ». Je dois cette indication à $D$. Cayer.

42. H. Dreyfus, op.cit. p. 5 I.

43. H. Dreyfus, « L'épiphénoménologie de Husserl », Etudes Philosophiques, I, 199I, p. 5860. 
perspective, la praxis, notre commerce quotidien avec les choses, n'est aucunement constitutive de notre relation au monde ${ }^{44}$. C'est précisément sur ce point que porte le débat qui l'oppose à Føllesdal ${ }^{45}$.

Føllesdal insiste sur le rôle que joue l'action dans notre relation au monde et il cherche à montrer que Husserl, et ce bien avant Heidegger, plus précisément à partir de igı6, accorde une place de plus en plus importante à l'activité pratique dans sa doctrine de la constitution. C'est ce que semblent confirmer plusieurs inédits et écrits posthumes, notamment Expérience et jugement ${ }^{46}$ où Husserl remarque que les choses de la nature n'épuisent pas le domaine du réel. Appartiennent aussi à celui-ci, les œuvres d'art, les livres et les constitutions d'État par exemple qui sont des « choses signifiantes, des objets de l'expérience ». Au sens objectif de ces objets, n'appartiennent pas seulement les déterminations qui originent de l'expérience doxique mais aussi celles qui

44. Dreyfus pourra toujours recourir à l'hypothèse de la destruction du monde par laquelle Husserl voulait faire valoir, dans les Idées directrices, la relativité de l'être à la conscience dans une perspective que d'aucuns qualifient d'idéaliste. Cependant, les remarques de Heidegger sur l'ontologie de la conscience au § I des Prolegomena semblent montrer que les déterminations d'être chez Husserl n'ont rien à voir avec la structure intentionnelle lig79: :145]. Comme l'indique R. Bernet dans son étude « Le monde et le sujet », Philosophie, 2I, 1989, P. 60, et comme Husserl le reconnaîtra lui-même au deuxième livre des Idées directrices, Paris, P.U.F., I982, « Le tort de "l'annihilation du monde" avait été justement de couper cette "corrèlation" entre le voir du monde et le monde vu. L hypothèse d'une "annihilation du monde" est donc contraire au sens de l'intentionnalité qui, sans cesse, porte le sujet à sintéresser aux choses du monde ainsi quäa leur apparaître. »

45. L'un des objets du litige qui opposait Dreyfus et Føllesdal au cours des rencontres de 1980 à Berkeley portait sur la possibilité de concevoir l'arrière-plan ou l'horizon des habiletés pratiques en termes d'intentionnalité. c'est-à-dire, pour Dreyfus, de croyances et de règles. Les textes de Dreyfus et de Føllesdal qui sont reproduit dans le présent numéro de Philosophiques poursuivent ce débat.

46. Voir E. Husserl, Expérience et jugement, tr. fr. D. Souche, Paris, P.U.F., 1970, § 65, voir également $\S 13$. Au deuxième livre des Idécs dircctriccs, Husscrl insiste sur l'indissociabilite de la personne et de son monde environnant [Umwelt] : " Ce monde environnant ne contient pas de simples objets, mais des choses d'usage l... des auvres d'art, des productions littéraires, des fournitures pour des actes religieux et juridiques [.J; et il ne contient pas seulement des personnes individuelles : les personnes sont bien plutôt des membres de communautés, d'unités personnelles d'un ordre plus élevé qui mènent leur vie propre en tant que totalités [.] 》, p. 257. 
se constituent par notre comportement volitif et axiologique [wertende].

Il appartient à leur sens objectif, au sens dans lequel ces choses nous sont données, et sont visées par nous, non seulement des déterminations issues de l'expérience doxique (la perception) ; mais elles portent aussi en soi des déterminations de sens qui renvoient à notre comportement axiologique et volontaire, et naissent de lui. Ce sont là aussi des déterminations que nous découvrons aux objets au sein de l'expérience; elles leur appartiennent réellement [real] en tant qu'ils sont des objets individuels, par exemple comme l'ustensilité d'un outil déterminé. Par ces déterminations, l'objet n'est assurément pas déterminé en ce qu'il est en soi et pour soi, mais en relation à nous, à notre appréciation et à notre vouloir, selon ce qu'il signifie pour nous ${ }^{47}$.

Mais la question est moins de savoir à qui revient le mérite en phénoménologie d'avoir mis l'accent sur l'activité pratique que sur l'aptitude d'une théorie de type husserlien de rendre compte de la dimension pratique sous-jacente au Dasein. Ainsi l'issue du débat porte sur l'intentionnalité :

Heidegger accepts intentional directedness as essential to human activity. but he denies that intentionality is mental, that it is, as Husserl (following Brentanol claimed, the distinguishing characteristic of mental states ${ }^{48}$.

Les objections de Dreyfus concernent donc à nouveau le mentalisme qu'il associe à l'intentionnalité chez Husserl et le problème du solipsisme qui en est issu. Comme je l'ai suggéré jusqu'à maintenant, il me semble que l'interprétation de Dreyfus va bien au-delà du jugement de Heidegger dans ses cours de Marbourg. Car, comme nous venons de le voir, une distinction très nette y est faite entre la découverte de Husserl, l'idée de corrélation, et l'interprétation supposément idéalisante des Idées directrices. Et on peut penser que c'est en ce sens qu'il lui est reproché de ne pas s'en être tenu à la radicalité du principe de la phénoménologie.

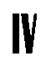

J'estime que là où Dreyfus a raison d'insister sur la véritable percée de Heidegger, c'est relativement au concept de transcendance. Il s'agit de la seconde thèse que Dreyfus attribue à Heidegger et suivant laquelle l'intentionnalité n'est qu'une forme de

47. Ibid., p. 321.

48. H. Dreyfus, op cit, p. 50-51. 
« transcendance ontique », c'est-à-dire qu'elle serait dérivée d'une forme plus originaire de transcendance que Heidegger appelle « l'être-au-monde ». Cette question mène directement au cœur de l'ontologie heideggerienne s'il est vrai que la thèse centrale de Sein und Zeit est que « Sein ist das transcendens schlechthin ${ }^{49}$. » Heidegger écarte d'emblée le sens épistémique et théologique de «transcendance » et Les problèmes fondamentaux de la phénoménologie posent que « le transcendant au sens strict, c'est le Dasein luimême $>^{50}$. On pourrait montrer que si la dimension ontologique à laquelle le concept de transcendance est supposé donner accès n'est pas de l'ordre de l'intentionnalité, celle-ci n'en constitue pas moins la «condition ontologique de possibilité ${ }^{5 \mathrm{I}}$. C'est ce que me semble énoncer ce passage lapidaire des Problèmes fondamentaux de la phénoménologie :

L'intentionnalité est la ratio cognoscendi de la transcendance. Cette dernière est la ratio essendi de l'intentionnalité dans ses différents modes $5^{2}$.

La « ratio cognoscendi » est la manière par laquelle se donne l'être de l'étant à la compréhension pré-conceptuelle et quotidienne. Ce mode de donation présuppose à son tour la constitution intentionnelle des comportements du Dasein. C'est pourquoi celle-ci constitue, me semble-t-il, la condition de possibilité de la transcendance. Maintenant, si la « ratio essendi » n'est ni de l'ordre du réel, ni du subjectif, comment y avons-nous accès ? Comment, en d'autres mots, peut-il devenir le thème d'une " ontologie scientifique » s'il n'est pas accessible de la même manière que le sont les étants dont il est pourtant indissociable ? Il faut, suggère Heidegger, que l'intentionnalité « devienne un problème $» 53$. Il

49. M. Heidegger, op.cit., p. 38. C'est ce que confirment un passage de la « Lettre sur l'humanisme » et de « Ce qui fait l'être-essentiel d'un fondement ou "raison" », Questions I, Paris Gallimard, 1968, p. I39 note I et p. 10I. Rappelons que la tâche assignée à la première partie de Sein und Zeit, et en particulier dans les $\$ \S$ 12, 13. 69, 83. est de foumir une « esquisse concrète » de la transcendance et de clarifier la « relation à » dans son essence primordiale. Voir également la remarque de Heidf:gger dans Metaphysische Anfangsgrüne der Logik im Ausgand von Leibniz, p. I64.

50. M. Heidegger, op. cit., p. 200.

51. Ibid., p. 90.

52. Op.cit., p. 80 .

53. M. Heidegger, « Ce qui fait l'être-essentiel d'un fondement ou "raison" », p. I48. 
faut une méthode qui reconduise le regard phénoménologique de l'appréhension de l'étant à la compréhension de l'être. Et cette méthode semble s'apparenter à la réduction phénoménologique de Husserl :

Pour Husserl, la réduction phénoménologique, telle qu'il l'a élaborée pour la première fois explicitement dans les Ideen de Igr3. est la méthode destinée à reconduire le regard phénoménologique de l'attitude naturelle de l'homme vivant dans le monde des choses et des personnes à la vie transcendantale de la conscience et à ses vécus noético-noématiques, dans lesquels les objets se constituent en tant que corrélats de la conscience. Pour nous, la réduction phénoménologique désigne la reconduction du regard phénoménologique de l'appréhension de l'étant - quelle que soit sa détermination - à la compréhension de l'être de cet étant (projet en direction de la modalité de son être-à-découvert) $)^{54}$.

\section{$V$}

Pour terminer, je voudrais résumer rapidement trois des points que j'ai cherché à clarifier à l'aide des cours de Marbourg. Le premier concerne l'originalité de la conception husserlienne de l'intentionnalité par rapport à celle de Brentano. Si Heidegger a pu parler d'une découverte de l'intentionnalité par Husserl, c'est qu'il a su la dissocier de la relation sujet / objet à laquelle Brentano l'avait subordonnée. Deuxièmement, malgré les nombreux reproches adressés à la doctrine husserlienne, Heidegger n'en retient pas moins l'idée de corrélation, c'est-à-dire le rôle que joue l'intentum ou le noème dans la caractérisation de la relation intentionnelle. Heidegger procède ensuite à un élargissement du champ de l'intentionnalitè en faisant valoir que la structure intentionnelle est sous-jacente à l'ensemble des comportements du Dasein. Cela présuppose, entre autres, que l'intentionnalité ainsi comprise soit apte à rendre compte de l'activité pratique. Le troisième point concerne la relation transcendance et intentionnalité. Nous avons tenté de montrer que si la dimension ontologique à laquelle le concept de transcendance est supposé donner accès n'est pas de l'ordre de l'intentionnalité, celle-ci n'en constitue pas moins la « condition ontologique de possibilité ». On pourrait même penser que le concept de «transcendance », le

54. M. Heidegger, Les Problèmes fondamentaux de la phénoménologie, op. cit., p. 39-40. 
concept par excellence de l'ontologie de Sein und Zeit, n'est en fait que le résultat d'une ontologisation de la structure intentionnelle que Husserl, par son cartésianisme, avait assujettie à la conscience.

Maintenant, pour être complet, cet exercise devrait ouvrir sur l'évaluation de l'apport pour la phénoménologie de la question du sens de être. Car c'est une chose de reconnaître le bien-fondé de la différence ontologique, c'en est une autre de déterminer son statut au sein de la phénoménologie. C'est ce dont témoignent les notes de Husserl en marge de son exemplaire de Sein und Zeit. J'estime qu'il faut voir dans ces notes autre chose que les remarques réprobatrices d'un maître vieillissant envers son élève. Car si Husserl a pu concevoir la phénoménologie heideggerienne de Sein und Zeit comme une «traduction » de sa propre «phénoménologie constitutive», il est vrai «amputée de sa fondation la plus profonde », c'est qu'il la croyait isomorphe à sa propre philosophie ${ }^{55}$.

Par ailleurs, il faudrait tenir compte du fait que le philosophe de Marbourg a presque complètement ignoré la phénoménologie husserlienne après les Idées directrices. Plusieurs observateurs avertis l'ont déploré ${ }^{56}$, notamment $P$. Ricoeur qui, soulignant l'importance de l'intentionnalité dite opérante [fungierendel dans Logique formelle et logique transcendantale ainsi que dans les Méditations cartésiennes, remarque que « cette intentionnalité opérante n'est pas sans parenté avec la transcendance de l'être-là selon Heidegger $\gg .57$ Les reproches de A. Kelkel dans son texte «Immanence de la conscience intentionnelle et transcendance

55. La phrase de Husserl est la suivante : « Heidegger transpose/transponiertl ou transuase Itransvasiertl'élucidation, par la phénoménologie constitutive, de toutes les régions de l'étant, et de l'étant universel, la région totale «monde», dans lordre de l'anthropologie. » J'emprunte ici la traduction de D. Souche-Dagues dans son article « La lecture husserlienne de Sein und Zeit », Philosophie, 21, 1989, p. Io.

56. R. Bernet, « Transcendance et intentionnalité : Heidegger et Husserl sur les prolégomènes d'une ontologie phénoménologique $»$, F. Volpi et al. (dir.) Heidegger et lídée de la phénoménologie, Dordrecht, Kluwer, 1989, p. 210 ; A. Marsulo, «"Sorge": Heideggers Verwandlung von Husserls Intentionalitätstruktur », dans C. Jamme et O. Poggeler (dir.), Phänomenologie im Widerstreit, Frankfurt, Suhrkamp, 1989 p. 247249 ; A. Kelkel « Immanence de la conscience intentionnelle et transcendance du Dasein ». F. Volpi et al. (dir.) Heidegger et lidée de la phénoménologie, Dordrecht, Kluwer, Ig89, p. $185^{-186 .}$ 
du Dasein » vont essentiellement dans le même sens. Selon lui, la lecture heideggerienne, que certains textes de Husserl semblent malgré tout autoriser, ne rend pas justice au développement et à l'approfondissement de cette notion dans l'cuuve husserlienne, et ce bien avant la Krisis. Je me permets de citer un long passage qui résume admirablement bien le problème :

Si tout au long des analyses critiques poursuivies par le philosophe de Marbourg bien des convergences comme des divergences se sont fait jour entre conscience intentionnelle et transcendance du Dasein, une dernière question mériterait de retenir notre attention : en quoi réside au juste le malentendu le plus grave au sujet de la théorie de l'intentionnalité entre Husserl et Heidegger? On répliquera sans doute que, plus fasciné par la phénoménologie des Logische Untersuchungen et des Ideen que par celle des Méditations cartésiennes ou de Formale und transzendentale Logik, il a méconnu la profondeur et toute l'ampleur de la « subjectivité de conscience » et la portée métaphysique de l'intentionnalité dite opérante, rabattant trop vite la themmatique intentionnelle sur le problème épistémologique traditionnel de la relation sujet-objet. N'est-ce pas méconnaître le sens profond de l'intentionnalité que de l'assimiler purement et simplement au « comportement à l'égard de l'étant »? Car ainsi réduite, elle ne saurait jouer son rôle que sur le fondement de la structure englobante de l'être-au-monde, qui devient de la sorte comme la ratio essendi de l'intentionnalité. Toutefois la fonction subordonnée qu'on lui confère correspond tout au plus à une interprétation psychologique qui a pu, un instant, être suggérée par les textes de Husserl. Mais à l'évidence, elle ne rend pas justice au développement et à l'approfondissement que cette notion clef connaît dans l'œuvre husserlienne, et ce bien avant la période de la Krisis. Si l'intentionnalité est comprise comme «Bewusstseinsleistung», embrassant les couches les plus profondes de la temporalisation et de la constitution de tout ètant, la critique heideggerienne ne risque-t-elle pas à la limite d'apparaître comme "impertinente ${ }^{5^{8}} »$ ?

A cet égard, une autre remarque de Husserl est importante, celleci en marge du paragraphe i3 dont la tâche était précisément de fournir une « esquisse concrète » de la transcendance et de clarifier la « relation à ${ }^{59}$, Husserl se demande :

57. P. Ricoeur, « Par-delà Husserl et Heidegger », Ies Cahiers de Philosophie, 7, 1989, P. 2122.

58. A. Kelkel, art. cit., p. 185-186.

59. Voir M. Heidegger, « Ce qui fait l'être-essentiel d'un fondement ou "raison" » op. cit., p. 101. 
Mais comment tout cela peut-il être éclairci, sinon par ma théorie de l'intentionnalité ? l... Ce qui est dit là, c'est ma propre théorie, simplement amputée de sa fondation la plus profonde $e^{60}$.

Schématiquement, on pourrait regrouper les remarques de Husserl en marge de Être et Temps autour des thèmes suivants : premièrement, n'est-il pas significatif que Husserl établisse à maintes reprises la correspondance entre sa théorie de l'intentionnalité et certains concepts clé de Êtreet Temps. Deuxièmement, en marge de la p. I6 où Heidegger indique que le mode d'accès et l'explicitation du Dasein doit se faire dans la « quotidienneté moyenne », Husserl mentionne ce qu'il a développé dans le deuxième volume des Idées directrices sous le titre de «psychologie intentionnelle de la personnalité ${ }^{6}$. Finalement, il y a cette remarque importante en marge de la p. $363 \mathrm{du}$ paragraphe $6 \mathrm{~g}$ intitulé « La temporalité de l'être-au-monde et le problème de la transcendance du monde $»$. On se rappellera tout d'abord cette note de Heidegger :

Que et comment l'intentionnalité de la « conscience » se fonde sinon à son tour dans la temporalité ekstatique du Dasein, c'est ce que montrera notre prochaine section ${ }^{62}$.

Voici maintenant la remarque de Husserl :

J'ai d'abord l'intentionnalité, et d'abord j'ai à l'interroger, à mettre en évidence la figure de son essence, et c'est ensuite que je peux voir que dans le « projet » de l'intentionnalité est inclus l'a priori, une figure essentielle qui, lorsque je l'ai constituée, peut par après être épistémologiquement directrice.

\section{Département de philosophie \\ Université du Québec à Montréal}

\footnotetext{
fo. Ibid., p. To.

6r. Husserl écrit : « Cest là, au sens qui est le mien, le chemin vers une psychologie intentionnelle de la personnalité au sens le plus large [...] », cité dans Souche-Dagues, art. cit., p. I9. Sur cette question, voir E. Husserl Idées directrices, vol. II, sections 48-64 et la discussion par Heidegger dans ses Prolegomena, p. 168 et sq, et l'excellent article de R. Bernet. « Le monde et le sujet », Philosophie, 21, 1989 .
}

62. M. Heidegger, Être et Temps, p. 252, note I. 